EESTI NSV TEADUSTE AKADEEMIA TOIMETISED. XIV KÖIDE

FOUSIKA-MATEMAATIKA- JA TEHNIKATEADUSTE SEERIA. 1965, NR. 2

ИЗВЕСТИЯ АКАДЕМИИ НАУК ЭСТОНСКОЙ ССР. ТОМ ХIV

СЕРИЯ ФИЗИКО-МАТЕМАТИЧЕСКИХ И ТЕХНИЧЕСКИХ НАУК. 1965, № 2

A. ЯГЕЛЬ

\title{
ХАРАКТЕРИСТИКА ОБЛАСТИ ПАРАМЕТРОВ ДОПУСТИМОСТИ ПРИ ОДНОМ КЛАССЕ ЗАДАЧ ПАРАМЕТРИЧЕСКОГО ЛИНЕЙНОГО ПРОГРАММИРОВАНИЯ
}

Статья посвящена изучению основной структуры множества параметров $t$, при которых существует допустимое решение следующей задачи: найти $\max \left[\left(c_{1}+t c\right) x_{1}+\right.$ $\vec{x}(t) \in \Omega_{t}$

$\left.+\sum_{j=2}^{n} c_{j} x_{i}\right]$, где $\Omega_{t}=\left\{\vec{x}(t):\left(\overrightarrow{a_{1}}+\overrightarrow{t d}\right) x_{1}+\sum_{j=2}^{n} \overrightarrow{a_{j}} x_{j}=\vec{b}+\overrightarrow{t b_{1}}, \vec{x} \geqslant \overrightarrow{0}\right\}$. Устанавливается, что множество $L_{t}=\left\{t: \Omega_{t} \neq \Theta\right\}$ не обязательно связное и замкнутое; множество $L_{t}$ однозначно определяется при помощи некоторых векторов $\vec{c}_{1}, \vec{c}_{2}, \vec{c}_{3}, \vec{c}_{4} \in R_{s}$, получение которых тесно связано с образующими определенного выпуклого многогранного конуса. Рассматривается вычисление образующих этого конуса.

\section{1. Постановка проблемы}

Параметрическая задача рассматриваемого класса ставится следующим образом: для каждого вещественного $t$ найти

$$
\begin{gathered}
\Phi(t)=\max \left[\left(c_{1}+c t\right) x_{1}+\sum_{j=2}^{n} c_{j} x_{j}\right], \\
\vec{x}(t) \in \Omega_{t} \\
\text { где } \Omega_{t}=\left\{\vec{x}^{t}(t):\left(\vec{a}_{1}+t \vec{d}\right) x_{1}+\sum_{j=2}^{n} \vec{a}_{j} x_{j}=\vec{b}+t \vec{b}_{1}, x \geqslant 0\right\},
\end{gathered}
$$

так что при фиксированном $t$ имеем задачу линейного программирования. До сих пор этот класс параметрических задач мало изучен. В данной статье исследуется структура множества параметров, при которых существует допустимое и оптимальное решение соответствующей задачи линейного программирования.

Введем следующие множества параметров: $L_{t}=\left\{t: \Omega_{t} \neq \Theta\right\}$, $O_{t}=\left\{t: t \in L_{t}, \Phi(t)<+\infty\right\}$ и $\mathbb{N}_{t}=\left\{t: \Psi_{t} \neq \boldsymbol{\Theta}\right\}$, где $\Theta$ обозначение пустого множества и $\Psi_{t}=\left\{\vec{x}(t):\left(\vec{a}_{1}+\overrightarrow{t d}\right) x_{1}+\sum_{j=2}^{n} \vec{a}_{j} x_{j}=\overrightarrow{0},\left(c_{1}+t c\right) x_{1}+\right.$ $\left.+\sum_{j=2}^{n} c_{j} x_{j}=1, \vec{x} \geqslant \overrightarrow{0}\right\}$. Назовем характеристикой множества $G \subset R$ $(R$ - множество вещественных чисел) минимальное число связных множеств, теоретико-множественной суммой которых является множество $G$; 
характеристику множества $G$ обозначим $H(G)$ и, если $G=\Theta$, то принимаем $H(G)=0$. Из теорем $1,3,4$ статьи [1] следует, что $H\left(N_{t}\right) \leqslant 1$ и $O_{t}=L_{t} \backslash N_{t}$ (теоретико-множественная разность двух множеств). Таким образом, основной интерес представляют множество $L_{t}$ и его характеристика. В статье ['] доказывается, что множество $N_{t}$ можно получить при помощи решения двух задач линейного программирования, поэтому в дальнейшем построению множества $O_{t}$ внимания не уделяется.

\section{2. Основные свойства множества $L_{t}$}

Пусть $R_{s}$ координатное пространство с размерностью $s$ и $\vec{c}_{i}(i=1,2$, $3,4)$ - векторы этого пространства. Определим следующие функции:

$$
f_{j}(t)= \begin{cases}0, & \text { если } j=0, \\ \frac{c_{j 3}+t c_{j 4}}{c_{j 1}+t c_{j 2}}, & \text { если } 1 \leqslant j \leqslant s \text { и } c_{j 1} \neq 0 \text { или } c_{j 2} \neq 0, \\ c_{j 3}+t c_{j 4}, & \text { если } 1 \leqslant j \leqslant s \text { и } c_{j 1}=c_{j 2}=0, \\ +\infty, & \text { если } j=s+1,\end{cases}
$$

где $c_{i i}-j$-ая координата вектора $\vec{c}_{i}$. Для $t \in R$ определим следующие множества, индексов:

$$
\begin{aligned}
& I_{t}^{+}=\left\{j: c_{j 1}+t c_{j 2}>0\right\} \cup\{s+1\}, \\
& I_{t}^{-}=\left\{j: c_{j 1}+t c_{j 2}<0\right\} \cup\{0\}, \\
& I_{t}^{0}=\left\{j: c_{j 1}=c_{j 2}=0\right\} .
\end{aligned}
$$

Если $I_{t}^{0}=\Theta$, то считаем, что $\min f_{j}(t)=1$. Множество решений уравнений $j \in I_{t}^{0}$

$c_{j 1}+c_{j 2} t=0, j \notin I_{t}^{0}$, обозначим $K_{t}$. Имеет место следующая

Теорема 1 (основная теорема). Для заданного $\left\{\Omega_{t}\right\}$ найдутся целое число s u векторы $\vec{c}_{i} \in R_{s}(i=1,2,3,4)$ такие, что множество $L_{t} \backslash K_{t}=\left\{t: \min f_{i}(t) \geqslant \max f_{i}(t), \min f_{j}(t) \geqslant 0\right\}$.

$$
j \in I_{t}^{+} \quad j \in I_{t}^{-} \quad j \in I_{t}^{0}
$$

Доказательство. По теореме $1\left[{ }^{2}\right]$ множество $\Omega_{t} \neq \Theta$ тогда и только тогда, когда при любом решении системы $A^{T}(t) \vec{u} \geqslant \overrightarrow{0}(T-$ транспонированная) имеет, место $\vec{u} \cdot \vec{b}(t) \geqslant 0$, где $\vec{b}(t)=\vec{b}+\overrightarrow{t b}_{1}$ и $A(t)=$

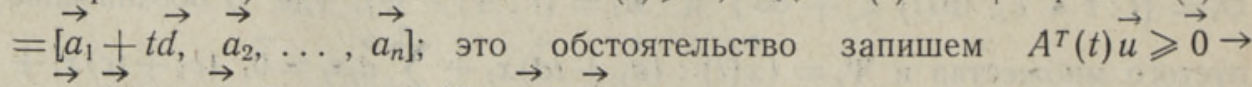
$\rightarrow \vec{u} \cdot \vec{b}(t) \geqslant \overrightarrow{0}$. Система $A^{T}(t) \vec{u} \geqslant \overrightarrow{0}$ в развернутом виде следующая:

$$
\left\{\begin{array}{l}
\vec{a}_{1}(t) \cdot \vec{u} \geqslant 0, \\
\overrightarrow{a_{i}} \cdot \vec{u} \geqslant 0, \quad i=2,3, \ldots, n .
\end{array}\right.
$$


По следствию 2 А статьи [ $\left.{ }^{3}\right]$ найдутся векторы $\vec{u}_{1}, \ldots, \vec{u}_{s}$ такие, что решением подсистемы $\vec{a}_{i} \vec{u} \geqslant 0, i=2,3, \ldots, n$, будет любой вектор $\vec{u}(\vec{\lambda})=$ $=\sum_{j=1}^{s} \lambda_{j} \vec{u}_{j}$, где $\vec{\lambda}=\left(\lambda_{1}, \ldots, \lambda_{s}\right) \geqslant \overrightarrow{0}$ и только те векторы. Подставляя $\overrightarrow{u(\lambda)}$ в неравенство $\vec{a}_{1}(t) \cdot \vec{u} \geqslant 0$, должно быть $\overrightarrow{a_{1}}(t) \cdot \vec{u}(\vec{\lambda}) \geqslant 0 \rightarrow \vec{u}(\vec{\lambda}) \cdot \vec{b}(t) \geqslant 0$. Обозначим $\overrightarrow{u_{j}} \cdot \vec{a}_{1}=c_{j 1}, \overrightarrow{u_{j}} \cdot \vec{d}=c_{j 2}, \overrightarrow{u_{i}} \cdot \vec{b}=c_{j 3}$ и $\vec{u}_{j} \cdot \vec{b}_{1}=c_{j 4}, j=1,2, \ldots, s$.

Следовательно,

$$
\left\{\begin{array}{rl}
\vec{\lambda} \cdot\left(\overrightarrow{c_{1}}+\vec{c}_{2} \cdot t\right) & \geqslant 0, \\
\vec{\lambda} & \geqslant \overrightarrow{0}
\end{array} \rightarrow \vec{\lambda} \cdot\left(\overrightarrow{c_{3}}+\overrightarrow{t c_{4}}\right) \geqslant 0\right.
$$

Если последнее обстоятельство не имеет места, то совместна система

$$
\left\{\begin{aligned}
\vec{\lambda}\left(\overrightarrow{c_{1}}+t \vec{c}_{2}\right) & \geqslant 0, \\
\vec{\lambda}\left(\overrightarrow{c_{3}}+t \overrightarrow{c_{4}}\right) & \leqslant-1, \\
\vec{\lambda} & \geqslant \overrightarrow{0} .
\end{aligned}\right.
$$

Для совместности системы (2) имеем (по теореме 1 статьи $\left.{ }^{2}\right]$ )

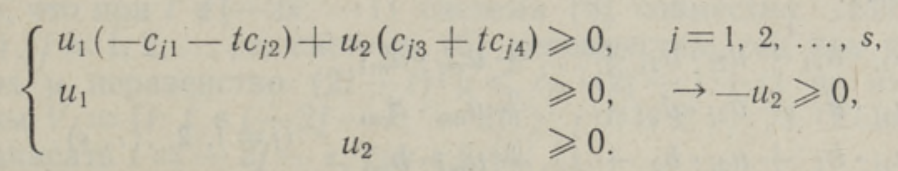

Пусть $\left(u_{1}, u_{2}\right)$ - решение системы (3); так как $u_{2} \geqslant 0$, то при $u_{2}>0$ $\rightarrow$ система $(2)$ несовместна, но тогда на множестве решений системы (1) $\vec{i} \cdot\left(\overrightarrow{c_{3}}+\overrightarrow{t c}_{4}\right) \geqslant 0$. Если в любом решении системы (3) координата $u_{2}=0$, то система (2) совместна, но тогда на множестве решений системы (1) $\vec{\lambda} \cdot\left(\overrightarrow{c_{3}}+t \overrightarrow{c_{4}}\right)<0$. Таким образом, необходимо, чтобы $u_{2}>0$. Обозначим $\frac{u_{1}}{u_{2}}=\mu$, следовательно, $\Omega_{t} \neq \Theta$ тогда и только тогда, когда совместна система

$$
\left\{\begin{aligned}
\mu \cdot\left(c_{j 1}+t c_{j 2}\right) & \leqslant c_{j 3}+t c_{j 4} \\
\mu & \geqslant 0 .
\end{aligned}\right.
$$

Если $j \in I_{t}^{0}$, то очевидно должно быть $c_{j 3}+t c_{j 4} \geqslant 0$, откуда для совместной системы (4) необходимо $\min f_{i}(t) \geqslant 0$. Если же кри $t_{0}$ имеем $j \in I_{t}^{0}$ $c_{i_{0} 1}+t_{0} c_{j_{0} 2}=0$ и $j_{0} \notin I_{t}^{0}$, то $t_{0} \in K_{t}$, следовательно, $t_{0} \notin L_{t} \backslash K_{t}$. Для индекса $j \in I_{t^{\prime}}^{0} \cup I_{t^{\prime}}^{-}$либо $\mu \geqslant f_{i}\left(t^{\prime}\right)$, либо $\mu \leqslant f_{j}\left(t^{\prime}\right)$. Из сказанного следует, что $L_{t} \backslash K_{t}=\left\{t: \min f_{i}(t) \geqslant \max f_{i}(t), \min f_{i}(t) \geqslant 0\right\}$. Теорема доказана.

$$
j \in I_{t}^{+} \quad j \in I_{t}^{-} \quad j \in I_{t}^{0}
$$


Граничную точку $t_{0}$ множества $L_{t}$ в дальнейшем называем критическим параметром этого множества.

След ств и е 1. Число критических параметров множества $L_{t}$ конечное.

Д о к а з а те л ь с т в о вытекает из вида функции $f_{j}(t)$ и из конечности множества $K_{t}$.

Следствие 2. Характеристика множества $L_{t}$ конечная.

Доказате льс т в о вытекает из следствия 1 .

Сл едст вие 3 . Если $t_{0}$ критический параметр иं $t_{0} \notin L_{t}$, то $t_{0} \in K_{t}$.

Доказательство. Пусть последовательность $\left\{t_{k}\right\}$ такая, что $t_{k} \in L_{t}$ и $\lim t_{k}=t_{0}$. Так как при любом $t_{k}$ система (4) совместна, то начи$k \rightarrow \infty$

ная с некоторого $k_{0}$ необходимо выполнение следующих условий: если $k \geqslant k_{0}$, то найдется индекс $j$ (не зависящий от $k$ ) такой, что $c_{j 3}+t_{k} c_{j 4}<0$, $c_{j 1}+t_{k} c_{j 2}<0, c_{j 3}+t_{0} c_{j 4}<0$ и $c_{j 1}+t_{0} c_{j 2}=0$. Из последних соотношений вытекает справедливость следствия.

Имеет место и обратная (к теореме 1)

Т ео рем а 2. Для любых $\vec{c}_{j} \in R_{s}, i=1,2,3,4$, можно поставить в соответствии $\left\{\Omega_{t}\right\}$ так, ито $\Omega_{t} \neq \Theta$ тогда и только тогда, когда $t \in L_{t}$, где $L_{t}$ множество параметров $t$, при которых совместна система (4).

Доказательство. Для определения векторов $\vec{a}_{1}, \vec{d}, \vec{b}, \vec{b}_{1}$ и $\vec{u}_{i}$ имеем систему

$$
\left\{\begin{array}{l}
c_{j 1}=u_{1 j} \cdot a_{11}+u_{2 j} \cdot a_{21}+\ldots+u_{m i} \cdot a_{m 1}, \\
c_{j 2}=u_{1 j} \cdot d_{1}+u_{2 j} \cdot d_{2}+\ldots+u_{m j} \cdot d_{m}, \\
c_{j 3}=u_{1 j} \cdot b_{1}+u_{2 j} \cdot b_{2}+\ldots+u_{m i} \cdot b_{m}, \\
c_{j 4}=u_{1 j} \cdot b_{11}+u_{2 j} \cdot b_{21}+\ldots+u_{m i} \cdot b_{m 1} .
\end{array} \quad(j=1,2, \ldots, s)\right.
$$

Одно из решений системы (5) следующее: $m=4 ; a_{11}=1, a_{i 1}=0 \quad(i=2$, $3,4) ; d_{2}=1, d_{i}=0,(i=1,3,4) ; b_{3}=1, b_{i}=0,(i=1,2,4) ; b_{11}=1, b_{i 1}=0$, $(i=1,2,3) ; u_{i j}=c_{j i}, \quad(i=1,2,3,4 ; j=1,2, \ldots, s)$. Обозначим $\vec{u}_{j}=\left(c_{i 1}\right.$, $\left.c_{j 2}, c_{j 3}, c_{j 4}\right)$. По следствию $4 \mathrm{~A}$ статьи [3] найдутся векторы $\vec{a}_{i} \in R_{4}$, $i=2,3, \ldots, p$, такие, что множество решений системы $\vec{a}_{i} \cdot \vec{u} \geqslant 0$, $i=2,3, \ldots, p$, совпадет со множеством $\{\vec{u}\}=\left\{\vec{u}: \sum_{j=1}^{s} \lambda_{j} \vec{u}_{j}=\vec{u}, \vec{\lambda} \geqslant \overrightarrow{0}\right\}$.

Если принимать $\Omega_{t}=\{\vec{x}(t): A(t) \vec{x}=\vec{b}(t), \vec{x} \geqslant \overrightarrow{0}\}$, где $\vec{b}(t)=\vec{b}+\vec{b}_{1}$, $A(t)=\left[\overrightarrow{a_{1}}+t \vec{d}, \vec{a}_{2}, \ldots, \vec{a}_{p}\right]$ и векторы $\vec{b}, \overrightarrow{b_{1}}, \vec{d}, \overrightarrow{a_{1}}$ суть решение системы (5), то, очевидно, $\Omega_{t} \neq \Theta$ тогда и только тогда, когда $t \in L_{t}$. Если же система (4) противоречива при любом $t \in R$, то и при любом $t \in R$ множество $\Omega_{t}=\Theta$. Теорема доказана.

Теоремы 1 и 2 , взятые вместе, дают, что с заданием четырех векторов $\vec{c}_{i}$, которые соответствуют данному множеству $\left\{\Omega_{t}\right\}$, полностью определяется множество $L_{t}$, т. е. его критические параметры, принадлежность их 
ко множеству $L_{t}$ и связные множества, теоретико-множественной суммой которых является множество $L_{t}$.

Для параметрических задач, изучаемых в статьях $[1,4,5]$, соответствующие множества обозначим $\Omega_{t}^{*}, N_{t}^{*}, L_{t}^{*}, O_{t}^{*}$ и $\Psi^{*}$; для этих задач имеем $H\left(N_{t}^{*}\right) \leqslant 1, H\left(L_{t}^{*}\right) \leqslant 1$ и $H\left(O_{t}^{*}\right) \leqslant H\left(N_{t}^{*}\right)+H\left(L_{t}^{*}\right) \leqslant 2$. Хотя при $\vec{b}_{1}=\overrightarrow{0}$ получаем в этих статьях рассмотренные задачи, но в данном случае далеко не всегда $H\left(L_{t}\right) \leqslant 1$, а можем утверждать только, что $H\left(O_{t}\right) \leqslant$ $\leqslant H\left(L_{t}\right)+1$. Тот факт, что строение множества $L_{t}$ может быть довольно сложное, подтверждается следующей

T ео ре мо й 3. Для любого натурального числа s существует множество $L_{t}$ такое, ито $H\left(L_{t}\right)=s+1$.

Доказ а тельство. Выбираем $\vec{c}_{i} \in R_{s}, i=1,2,3,4$, такие, что для каждого $j=1,2, \ldots, s$, выполняются следующие условия: $c_{j 1}=2 j, c_{j 2}=1$, $c_{j 4}>0, \frac{c_{j 3}}{c_{j 4}}=2 j-1$. Тогда система (4) принимает вид

$$
\left\{\begin{aligned}
(2+t) \mu & \leqslant c_{14}(1+t), \\
(4+t) \mu & \leqslant c_{24}(3+t), \\
\ldots \ldots & \ldots \\
(2 s+t) \mu & \leqslant c_{s 4}(2 s-1+t), \\
\mu & \geqslant 0 .
\end{aligned}\right.
$$

Очевидно, что при $t \notin[-2 s ;-1)$ система (6) совместна. Для $t \in(-2 j$; $+2 j+1), j=1,2, \ldots, s$, система (6) несовместна, так как при неотрицательных $\mu$ неравенство $(2 j+t) \cdot \mu \leqslant c_{j 4} \cdot(2 j-1+t)$. не имеет места. Обозначим $V_{j}=\{t: t \in(-2 j-1 ;-2 j)\}, j=1,2, \ldots, s$. Если $t \in V_{j}$, то можем записать $t=-2 j-\varepsilon$, где $\varepsilon \in(0 ; 1)$.

Пусть интервал $V_{j}$ фиксирован; тогда неравенства системы (6) запишем в виде $[2(j-k)+t] \cdot \mu \leqslant c_{i-k, 4} \cdot[2(j-k)-1+t], k=j-s$, $\ldots, j-1$. Имеем $2(j-k)+t=-2 k-\varepsilon$ и $2(j-k)-1+t=-2 k-$ $-1-\varepsilon$. Так как для интервала $V_{i}$ множество $I_{t}^{0}=\Theta$, то для определения $c_{j 4}$ на этом интервале имеем условие

$$
\min _{j-s \leqslant k \leqslant-1} \frac{c_{j-k, 4} \cdot[2(j-k)-1+t]}{2 \cdot(j-k)+t} \geqslant \max _{0 \leqslant k \leqslant j-1} \frac{c_{j-k, 4} \cdot[2(j-k)-1+t]}{2 \cdot(j-k)+t}
$$

или

$$
\min _{j-s \leqslant k \leqslant-1} \frac{c_{j-k, 4} \cdot(-2 k-1-\varepsilon)}{-2 k-\varepsilon} \geqslant \max _{0 \leqslant k \leqslant j-1} \frac{c_{j-k, 4} \cdot(-2 k-1-\varepsilon)}{-2 k-\varepsilon} .
$$

Е.ли для некоторого $\varepsilon \in(0 ; 1)$ минимум достигается при индексе $k=-1$ и максимум при $k=0$, то имеем

$$
c_{j+1,4}\left(1+\frac{1}{\varepsilon-2}\right) \geqslant c_{j, 4}\left(1+\frac{1}{\varepsilon}\right) .
$$

Наоборот, если $c_{j 4}(j=1,2, \ldots, s)$ удовлетворяют условию (7) для некоторого $\varepsilon \in(0 ; 1)$, то независимо от $j$ минимум 
и максимум достигаются на индексах $k=-1$ и соответственно $k=0$. Это вытекает из того, что можем выбирать $c_{j+1,4}(j \leqslant s-1)$ так, что с увеличением $k$ величина $1+\frac{1}{2 k+\varepsilon}$ уменьшается, а с уменьшением $k$ эта величина увеличивается. Пусть $\overrightarrow{c_{4}}$ фиксирован и при некотором $\varepsilon \in(0 ; 1)$ условия (7) выполнены; покажем, что множество параметров на интервале $V_{j}(j=1,2, \ldots, s-1)$ связное, т. е. множество параметров, которые удовлетворяют условию (7). Из условий (7) имеем, что $\frac{c_{j+1,4}}{c_{j, 4}} \geqslant 1+\frac{2}{\varepsilon-\varepsilon^{2}}=g(\varepsilon)$. Пусть $\min _{1 \leqslant j \leqslant s-1} \frac{c_{i+1,4}}{c_{j, 4}}=\frac{c_{j_{0}+1,4}}{c_{j_{0}, 4}}=c_{0}$, следовательно, $g(\varepsilon) \leqslant c_{0}$. Очевидно, что $\min g(\varepsilon)=g\left(\frac{1}{2}\right)$; левее и правее $\varepsilon \in(0 ; 1)$

от точки $\varepsilon=\frac{1}{2}$ функция $g(\varepsilon)$ на интервале $(0 ; 1)$ строго возрастающая, отсюда вытекает связность соответствующего множества параметров, которые обозначим $S_{i}(j=1,2, \ldots, s-1)$. Пусть $S_{0}=\{t: t \geqslant-1\}$ и $S_{s}=\{t: t<-2 s\}$. Применяя теорему 2 , можем построить $\left\{\Omega_{t}\right\}$ так, что соответствующее $L_{t}=\bigcup_{j=0}^{s} S_{j}$. Из доказанного вытекает, что $H\left(L_{t}\right)=$ $=\sum_{j=0}^{s} H\left(S_{j}\right)=s+1$. Теорема доказана.

Является ли полученный результат наихудшим в том смысле, что при любых $\vec{c}_{i} \in R_{s}(i=1,2,3,4)$ для соответственного множества $L_{t}$ имеем $H\left(L_{t}\right) \leqslant s+1$, пока не известно. Ответ можем дать только в частном случае, именно справедлива следующая

Теорем а 4. Eсли $\vec{c}_{i} \in R_{s}(i=1,2,3,4)$ такие, что $\vec{c}_{2}>\overrightarrow{0}$ u $D_{j}>0$ $(j=1,2, \ldots, s)$, то $H\left(L_{t}\right) \leqslant s+1$, где $D_{j}=\left|\begin{array}{ll}c_{j 1} & c_{j 2} \\ c_{j 3} & c_{j 4}\end{array}\right|$.

Д оказ а тельст в о. Детальное доказательство опускаем, а приводим только его идею. Так как $\frac{d f_{j}(t)}{d t}=\frac{D_{j}}{\left(c_{j 1}+t c_{j 2}\right)^{2}}(j=1,2, \ldots, s)$, то по условиям теоремы функции $f_{j}(t)$ неубывающие и нелинейные. Обозначим решение уравнения $c_{j 1}+t c_{j 2}=0$ через $t_{j}$. В области $\left(-\infty ; t_{i}\right)$ функция $f_{j}(t)$ выпуклая и в области $\left(t_{j} ;+\infty\right)$ - вогнутая. Можем считать, что $t_{j} \leqslant t_{j+1}(j=1,2, \ldots, s-1)$. Рассмотрим непустой интервал $\left(t_{j_{0}} ; t_{j_{0}+1}\right)$. Легко видеть, что на этом интервале при $j \geqslant j_{0}+1$ имеем $j \in I_{t}^{-}$и при $j \leqslant j_{0}$ $j \in I_{t}^{+}$. Из сказанного следует, что на интервале $\left(t_{j_{0}} ; t_{j_{0}+1}\right) \quad \pi(t)=$ $=\max f_{j}(t)$ выпуклая функция и $\gamma(t)=\min f_{j}(t)$ вогнутая функция. $j \in I_{t}^{-}$ $j \in I_{t}^{+}$

Отсюда следует, что область параметров $t$, при которых $\gamma(t)-\pi(t) \geqslant 0$. связная. Число интервалов $\left(t_{j} ; t_{j+1}\right) s-1$, и при достаточно больших it| $\gamma(t)-\pi(t) \geqslant 0$, следовательно, $H\left(L_{t}\right) \leqslant s+1$. Теорема доказана.

Легко убедиться, что такая идея доказательства приложима при условиях $\vec{c}_{2} \geqslant \overrightarrow{0}$ и $D_{j} \geqslant 0(j=1,2, \ldots, s)$ или $\vec{c}_{2} \leqslant \overrightarrow{0}$ и $D_{j} \leqslant 0(j=1,2, \ldots, s)$. В общем случае функции $\pi(t)$ и $\gamma(t)$ не обладают требуемыми свойствами выпуклости и вогнутости, в чем и заключается трудность распространения теоремы 4 на все параметрические задачи рассматриваемого класса. 


\section{3. Вычисление векторов $\vec{c}_{i}$}

Во многих приложениях представляет интерес фактическое нахождение векторов $\overrightarrow{c_{i}}(i=1,2,3,4)$, которые соответствуют заданной параметрической задаче. Построение соответствующего множества $L_{t}$ при помощи этих векторов уже под силу современным быстродействующим вычислительным машинам. Ниже мы укажем на одну возможность вычисления векторов $\overrightarrow{c_{i}}(i=1,2,3,4)$, которая в некоторых случаях применима.

При доказательстве теоремы 1 мы пользовались следствием 2А статьи [3], согласно которому найдутся векторы $\vec{u}_{1}, \ldots, \vec{u}_{s}$ такие, что решением системы $\vec{a}_{i} \cdot \vec{u} \geqslant 0, i=1,2, \ldots, n$, будут векторы $\vec{u}(\vec{\lambda})=\sum_{j=1}^{s} \lambda_{j} \vec{u}_{j}, \lambda_{1} \geqslant \overrightarrow{0}$ $\lambda_{1}, \ldots, \lambda_{s} \geqslant 0$ и только эти векторы. Оказывается, что существует очень простой и хорошо поддающийся программированию алгоритм вычисления векторов $\vec{u}_{1}, \ldots, \vec{u}_{s}$. Из доказательства теоремы 1 видно, что при фактическом знании векторов $\overrightarrow{u_{j}}, j=1,2, \ldots, s$, получение векторов $\overrightarrow{c_{i}}, i=1,2$, 3,4 уже не представляет труда.

Множество векторов $\vec{u}_{j}$ для системы $\overrightarrow{a_{i}} \cdot \vec{u} \geqslant 0(i=1, \ldots, n \leqslant s)$, которые удовлетворяют следствию $2 \mathrm{~A}$ статьи [ $\left.{ }^{3}\right]$, обозначим $C_{n}$. Определяем

$$
\begin{aligned}
& U_{n}^{+}=\left\{\overrightarrow{u_{j}}: \vec{u}_{j} \in C_{n}, \vec{u}_{j} \cdot \vec{a}_{n+1}>0\right\}, \quad U_{n}^{0}=\left\{\overrightarrow{u_{j}}: \vec{u}_{j} \in C_{n}, \vec{u}_{j} \cdot \vec{a}_{n+1}=0\right\}, \\
& U_{n}^{-}=\left\{\overrightarrow{u_{j}}: \overrightarrow{u_{j}} \in C_{n}, \overrightarrow{u_{j}} \cdot \vec{a}_{n+1}<0\right\} \quad \text { и } \quad U_{n}=\left\{\vec{u}_{i j}: \vec{u}_{i j}=\lambda_{i} \vec{u}_{i}+\lambda_{j} \vec{u}_{j},\right. \\
& \left.\lambda_{i}+\lambda_{j}=1, \quad \lambda_{i} \geqslant 0, \quad \lambda_{j} \geqslant 0, \quad \vec{u}_{i j} \cdot \vec{a}_{n+1}=0, \quad \vec{u}_{i} \in U_{n}^{+}, u_{j} \in U_{n}^{-}\right\} .
\end{aligned}
$$

Имеет место следующая

Теорема 5 .

$$
C_{n+1}=\left\{\begin{array}{lllll}
U_{n}^{+} \cup U_{n}^{0} \cup U_{n} & , \text { eсли } U_{n}^{+} \neq \Theta \text { u } U_{n}^{-} \neq \Theta, \\
C_{n} & , \text { ссли } U_{n}^{-}=\Theta \text { или } C_{n}=U_{n}^{0}, \\
\overrightarrow{0}\} & , \text { если } U_{n}^{+}=\Theta \text { и } U_{n}^{0}=\Theta .
\end{array}\right.
$$

Доказательство. Интерес представляют случаи $U_{n}^{+} \neq \Theta$ и $U_{n}^{-} \neq \Theta$, при остальных условиях доказательство тривиальное. Пусть $A<$ выпуклый конус, образующие которого суть элементы конечного множества $A$. Из определения множеств $U_{n}^{+}, U_{n}^{0}, U_{n}$ следует, что $\left(U_{n}^{+} \cup U_{n}^{0} \mathrm{U}\right.$ $\left.\mathrm{U} U_{n}\right)<\subset\left(C_{n+1}\right)<$. Докажем обратное включение указанных множеств. Так как $\left(C_{n+1}\right)^{<} \subset\left(C_{n}\right)^{<}$, то $\vec{u}_{0} \in\left(C_{n}\right)^{<}$. Если $\overrightarrow{u_{0}} \in\left(U_{n}^{+} \cup U_{n}^{0}\right)^{<}$, то очевидно $\vec{u}_{0} \in\left(U_{n}^{+} \cup U_{n}^{0} \cup U_{n}\right)<$. По определению $\left(C_{n}\right)^{<}$имеем $\vec{u}_{0}=$ $=\sum_{\vec{u}_{i} \in U_{n}^{+} \cup U_{n}^{0}} \lambda_{i}^{(0)} \vec{u}_{i}+\sum_{\vec{u}_{k} \in U_{n}^{-}} \gamma_{k}^{(0)} \vec{u}_{k}, \vec{\lambda}^{(0)} \geqslant \overrightarrow{0}, \vec{\gamma}^{(0)} \geqslant \overrightarrow{0}$. Если $\vec{u}_{0} \notin\left(U_{n}^{+} \cup U_{n}^{0}\right)^{<}$, 
то должен быть $\vec{\lambda}^{(0)} \neq \overrightarrow{0}$ и $\vec{\gamma}^{(0)} \neq \overrightarrow{0}$, так как $\vec{a}_{n+1} \cdot \vec{u}_{0} \geqslant 0$; следовательно, векторы $\vec{z}=2 \underset{\vec{u}_{i} \in U_{n}^{+} \cup U_{n}^{-}}{ } \lambda_{i}^{(0)} \vec{u}_{i}$ и $\vec{y}=2 \sum_{\vec{u}_{k} \in U_{n}^{-}} \gamma_{k}^{(0)} \vec{u}_{k}$ не равны, причем $\vec{z}, \vec{y} \in\left(C_{n}\right)<$.

Найдется $\lambda^{\prime} \epsilon(0 ; 1)$ такое, что $\vec{a}_{n+1} \cdot\left[\lambda^{\prime} \vec{z}+\left(1-\lambda^{\prime}\right) \vec{y}\right]=0$, откуда $\quad \lambda^{\prime}=$ $=\frac{\vec{a}_{n+1} \cdot \vec{y}}{\rightarrow \rightarrow}$. Пусть $\left[\vec{x}_{1} ; \vec{x}_{2}\right]=\left\{\vec{x}: \vec{x}=\lambda \vec{x}_{1}+(1-\lambda) \vec{x}_{2}, \quad \lambda \in[0 ; 1]\right\}$. $a_{n+1} \cdot y-a_{n+1} \cdot z$

Обозначим $\vec{z}_{1}=\lambda^{\prime} \vec{z}+\left(1-\lambda^{\prime}\right) \vec{y}$, из сказанного следует, что $\vec{u} 0 \in\left[\vec{z} ; \vec{z}_{1}\right] \subset$ $\subset[\vec{z} ; \vec{y}]$, следовательно, $\vec{u}_{0}=\mu^{\prime} \vec{z}+\left(1-\mu^{\prime}\right) \overrightarrow{z_{1}}$, где $\mu^{\prime} \in[0 ; 1]$. Очевидно, что $1-\lambda^{\prime}=\frac{\vec{a}_{n+1} \cdot \vec{z}}{\vec{a}_{n+1} \cdot \vec{z}-\vec{a}_{n+1} \cdot \vec{y}} \cdot$ Подставляя и вычисляя, имеем $\overrightarrow{z_{1}}=\lambda^{\prime} \vec{z}+$

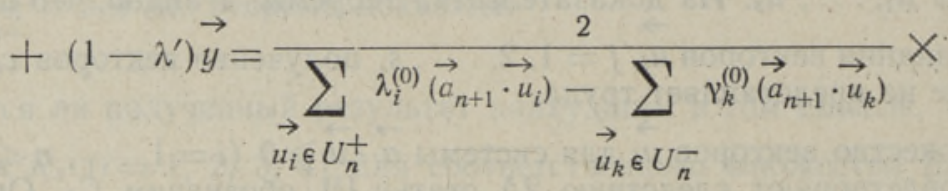

$$
\begin{aligned}
& \times\left[\sum_{\vec{u}_{i} \in U_{n}^{+}, \vec{u}_{k} \in U_{n}^{-}}\left(\lambda_{i}^{(0)} \gamma_{k}^{(0)}\left(-\vec{a}_{n+1} \cdot \vec{u}_{k}\right) \vec{u}_{i}+\gamma_{k}^{(0)} \lambda_{i}^{(0)}\left(\vec{a}_{n+1} \cdot \vec{u}_{i}\right) \vec{u}_{k}\right)\right] .
\end{aligned}
$$

Из соотношения (8) легко видно, что $\vec{u}_{0} \in\left(U_{n}^{+} \cup U_{n}^{0} \cup U_{n}\right)<$, следовательно, $\left(C_{n+1}\right)^{<} \subset\left(U_{n}^{+} \cup U_{n}^{0} \cup U_{n}\right)^{<}$. Теорема доказана.

Хотя вычисление векторов множества $U_{n}$ очень простое, но правило построения множества $C_{n+1}$ на основе теоремы 5 практически не всегда применимо из-за громоздкой численности множества $U_{n}$; тем более, что множество $U_{n+1}$ строится исходя из множества $C_{n+1}$. Этот недостаток вызван тем, что множество $U_{n}^{0} \cup U_{n} \cup U_{n}^{+}$содержит неэкстремальные образующие (определение см. в [3]) конуса $\left(C_{n+1}\right)^{<}$, которые совершенно лишние.

Если матрица $A$ системы $\overrightarrow{a_{i}} \cdot \vec{u} \geqslant 0(i=1,2, \ldots, n)$ квадратичная, то может быть применима следующая

Т е ор е м а 6 . Пусть $n$ ранг системы $\vec{a}_{i} \cdot \vec{u} \geqslant 0(i=1,2, \ldots, n)$ u соответствующая обратная матрица $A^{-1}$, тогда $C_{n}=\left\{\vec{a}_{1}^{\prime} ; \ldots ; \vec{a}_{n}^{\prime}\right\}$, где $\vec{a}_{i}^{\prime} i$-тый столбец матрицы $A^{-1}$.

Доказательство. Пусть $\vec{u}_{0}$ такое, что $A \vec{u}_{0}=\vec{y}_{0} \geqslant \overrightarrow{0}$, тогда $\overrightarrow{u_{0}}=A^{-1} \vec{y}_{0}$. Если же $\overrightarrow{y^{*}} \geqslant \overrightarrow{0}$, то $\overrightarrow{u^{*}}=A^{-1} \vec{y}^{*}$ является решением системы $A \vec{u} \geqslant \overrightarrow{0}$. Так как $A^{-1} \vec{y}_{0}=\sum_{i=1}^{n} y_{i}^{(0)} \vec{a}_{i}^{\prime}$, то из произвольности $\vec{y}_{0} \geqslant \overrightarrow{0}$ следует справедливость теоремы. Теорема доказана. 
Практически вычисления покажут, что теорему 6 целесообразно в первую очередь применять для квадратичной подсистемы $\vec{a}_{i} \cdot \vec{u} \geqslant 0$, $i=1,2, \ldots, m$, а затем применить теорему 5 .

Если число переменных больше числа неравенств в системе $\vec{a}_{i} \cdot \vec{u} \geqslant 0$, $i=1,2, \ldots, n$, то построение множества $C_{n}$ придется начинать с образующих конуса $\left(C_{1}\right)^{<}$. Для построения конуса $\left(C_{1}\right)<$ можем поступить следующим образом: $\vec{u}_{1}, \ldots, \vec{u}_{m-1}$ являются базисом подпространства $\overrightarrow{a_{1}} \cdot \vec{u}=0, \vec{u}_{m}=-\sum_{i=1}^{m-1} \vec{u}_{i}, \vec{u}_{m+1}=\vec{a}_{1}$ и принимаем $C_{1}=\left\{\vec{u}_{1} ; \ldots ; \vec{u}_{m+1}\right\}$. Легко видеть, что $\left(C_{1}\right)<=\left\{\vec{u}: \overrightarrow{a_{1}} \cdot \vec{u} \geqslant 0\right\}$. Для построения конусов $\left(C_{2}\right)^{<}$, $\left(C_{3}\right)^{<}, \ldots$ можно уже пользоваться теоремой 5. Если множество образующих $C_{n}$ получено, то вычисление векторов $\vec{c}_{i}, i=1,2,3,4$, не представляет особого труда, это видно из доказательства теоремы 1 .

В заключение отметим, что в связи с вычислительным процессом интерес представляет следующий вопрос: как удалить при помощи приемлемого объема вычислений векторы множества $U_{k}^{0} \cup U_{k}$, которые удовлетворяют условию $\vec{u}_{i_{0}}=\sum \alpha_{i} \vec{u}_{i}, \alpha_{i} \geqslant 0$ ? От успешного решения именно этого

$$
\begin{gathered}
\vec{u}_{i} \in U_{k}^{0} \cup U_{k} \\
i \neq i_{0}
\end{gathered}
$$

вопроса зависит успех применения алгоритма, основанного на теореме 5 .

\section{ЛИТЕРАТ У Р А}

1. Ягель А. Ю., Тр. Ин-та физ. и астрон. АН ЭССР, № 24, 46 (1964).

2. Фа н ь Цз и, О системах линейных неравенств (сб. переводов), М., 1959, 215-262.

3. Голдм ан А. Дж., Т акке р А. У., Многогранные выпуклые конусы (сб. переводов), М., 1959, 142-161.

4. Я гель А. Ю., Изв. АН ЭССР. Сер. фнз.-мат. и техн. наук, № 4, $382-402$ (1964).

5. К а р а бе го в В.-К. И., Ж. вычислит. мат. и матем. физики, 3, № 3, $547-558$ (1963).

Ннститут физики и астрономии

Академии наук Эстонской ССР

Поступила в редакцию 22/VII 1964

\section{A. JÄGEL}

\section{LUBATAVATE PARAMEETRITE PIIRKONNA KARAKTERISTIKA OHE KLASSI PARAMEETRILISTE LINEAARSETE PROGRAMMEERIMISULESANNETE PUHUL}

Käesolevas artiklis uuritakse parameetrite $t$ hulgas struktuuri, millest sôltub

$$
\Phi(t)=\underset{\vec{x}(t) \in \Omega_{t}}{\max }\left[\left(c_{1}+t c\right) x_{1}+\sum_{j=2}^{n} c_{j} x_{i}\right],
$$

kus

$$
\left.\Omega_{t}=\vec{x}(t):\left(\overrightarrow{a_{1}}+t \vec{d}\right) x_{1}+\sum_{j=2}^{n} \vec{a}_{i} x_{j}=\vec{b}+\overrightarrow{t b_{1}}, \vec{x} \geqslant \overrightarrow{0}\right\}
$$


Tõestatakse, et hulk $L_{t}=\left\{t: \Omega_{t} \neq \Theta\right\}$ ei tarvitse olla sidus ega kinnine. Hulk $L_{t}$ määratakse üheselt teatud vektorite $\overrightarrow{c_{1}}, \overrightarrow{c_{2}}, \overrightarrow{c_{3}}, \overrightarrow{c_{4}} \in R_{s}$ abil, mille saamiseks tuleb arvutada parameetrilise ülesandega seotud koonuse moodustajad. Vaadeldakse selle koonuse moodustajate faktilist leidmist.

\section{A. $J \ddot{A} G E L$}

\section{CHARACTERISTICS OF THE REGION OF THE PARAMETER OF ADMISSIBILITY IN A PROBLEM OF PARAMETRIC LINEAR PROGRAMMING}

In the present paper the essential structure of the set of real parameter $t$ is described in case of which there exists an admissible solution: it is necessary to find $\Phi(t)=\max$
$\vec{x}(t) \in \Omega_{t}$$\left[\left(c_{1}+t c\right) x_{1}+\sum_{j=2}^{n} c_{j} x_{j}\right]$ where $\Omega_{t}=\left\{\vec{x}(t): \overrightarrow{\left(a_{1}+t d\right)} x_{1}+\sum_{j=2}^{n} \overrightarrow{a_{j}} x_{j}=\vec{b}+t \vec{b}_{1}, \vec{x} \geqslant \overrightarrow{0}\right\}$. It is established that the set $L_{t}=\left\{t: \Omega_{t} \neq \Theta\right\}$ is not always connected and closed. The set $L_{t}$ is solely determined by means of certain vectors $\overrightarrow{c_{1}}, \overrightarrow{c_{2}}, \overrightarrow{c_{3}}, \overrightarrow{c_{4}}$. The calculation of these vectors is described. 